\title{
Prioritization of non-coding disease-causing variants and long non-coding RNAs in liver cancer
}

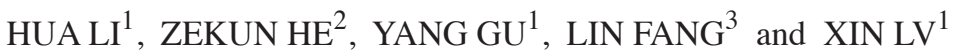 \\ ${ }^{1}$ Department of Anesthesiology, Shanghai Pulmonary Hospital, Tongji University School of Medicine, Shanghai 200433; \\ ${ }^{2}$ Department of Clinical Medicine, Fuzhou Medical College of Nanchang University, Fuzhou, Jiangxi 344000; \\ ${ }^{3}$ Department of Thyroid and Breast Surgery, Shanghai Tenth People's Hospital, \\ Tongji University, School of Medicine, Shanghai 200072, P.R. China
}

Received March 20, 2015; Accepted June 16, 2016

DOI: $10.3892 / \mathrm{ol} .2016 .5135$

\begin{abstract}
There are multiple bioinformatics tools available for the detection of coding driver mutations in cancers. However, the prioritization of pathogenic non-coding variants remains a challenging and demanding task. The present study was performed to discriminate non-coding disease-causing mutations and prioritize potential cancer-implicated long non-coding RNAs (lncRNAs) in liver cancer using a logistic regression model. A logistic regression model was constructed by combining 19,153 disease-associated ClinVar and human gene mutation database pathogenic variants as the response variable and non-coding features as the predictor variable. Genome-wide association study (GWAS) disease or trait-associated variants and recurrent somatic mutations were used to validate the model. Non-coding gene features with the highest fractions of load were characterized and potential cancer-associated IncRNA candidates were prioritized by combining the fraction of high-scoring regions and average score predicted by the logistic regression model. H3K9me3 and conserved regions were the most negatively and positively informative for the model, respectively. The area under the receiver operating characteristic curve of the model was 0.92 . The average score of GWAS disease-associated variants was significantly increased compared with neutral single nucleotide polymorphisms (5.8642 vs. 5.4707; $\mathrm{P}<0.001)$, the average score of recurrent somatic mutations of liver cancer was significantly increased compared with non-recurrent somatic mutations (5.4101 vs. 5.2768; $\mathrm{P}=0.0125)$. The present study found regions in lncRNAs and introns/untranslated regions of protein coding genes where mutations are most
\end{abstract}

Correspondence to: Mr. Xin Lv, Department of Anesthesiology, Shanghai Pulmonary Hospital, Tongji University School of Medicine, 507 Zheng Min Road, Yangpu, Shanghai 200433, P.R. China

E-mail: 18621710790@163.com

Key words: liver cancer, non coding genome, pathogenic variant, logistic regression model likely to be damaging. In total, 847 lncRNAs were filtered out from the background. Characterization of this subset of lncRNAs showed that these IncRNAs are more conservative, less mutated and more highly expressed compared with other control lncRNAs. In addition, 23 of these lncR NAs were differentially expressed between 12 pairs of liver cancer and adjacent normal specimens. The logistic regression model is a useful tool to prioritize non-coding pathogenic variants and lncRNAs, and paves the way for the detection of non-coding driver lncRNAs in liver cancer.

\section{Introduction}

The wide application of next-generation sequencing has identified millions of somatic alterations in cancer genomes (1). Certain alterations responsible for oncogenesis are termed driver mutations, but the majority remain passenger mutations, which accumulate and have little function in cancer progression (2). At present, there are numerous bioinformatics tools available on driver mutation prediction; the tools mostly focus on coding mutations that change the amino acid residues, for example the sorting tolerant from intolerant algorithm (3) and polymorphism phenotyping tool (4). By contrast, there are few studies conducted on the evaluation of the functional impact of non-coding variants, and identification of non-coding drivers in a typical tumor is a challenging and unsolved problem.

Recently, the interpretation of non-coding variants has been achievable due to the production of high-throughput projects, such as the Encyclopedia of DNA Elements (ENCODE) Consortium (5) and the US National Institutes of Health Roadmap Epigenomics project (6). Based on these data, a number of tools have been developed to annotate potential regulatory variants or suggest the most likely damaging variants, such as RegulomeDB (7), HaploReg (8) and Funseq (9). Despite the high efficiency of functional annotation of non-coding variants with these tools, there have been certain criticisms of the empirical scoring algorithms, such as lack of accuracy and specificity (10). Recently, machine-learning models were introduced and trained on pathogenic variants or nearly-fixed/fixed human derived alleles to better predict and score the functionalities of non-coding variants $(11,12)$. 
Fu et al (13) reported a computational framework, FunSeq2, that combines an adjustable data context integrating large-scale genomics, such as 1000 Genomes and ENCODE data, and cancer resources with a weighted scoring system. Variants are scored by combining inter- and intra-species conservation, loss- and gain-of-function events for transcription-factor binding, enhancer-gene linkages and network centrality, and per-element recurrence across samples (13). Kircher et al (11) contrasted the annotations of fixed or nearly-fixed derived alleles in humans with those of simulated variants and developed combined annotation-dependent depletion (CADD). CADD, as a trained support vector machine, measures deleteriousness, which may be measured systematically across the genome assembly (14). Implementation of CAAD successfully differentiated 14.7 million high-frequency human-derived alleles from 14.7 million simulated variants.

Long non-coding RNAs (lncRNAs) are a class of mRNA-like transcripts ranging between $200 \mathrm{bp}$ and $100 \mathrm{~kb}$ in size. lncRNAs lack significant open-reading frames and are not translated into proteins. There has been a large number of studies that reported lncRNAs to be involved in a wide range of physiological processes by regulating gene expression at various levels, including chromatin architecture, transcription, RNA splicing, and protein translation and turnover (15-19). Previously, the role of lncRNAs as drivers of tumor suppressive and oncogenic functions has been reported in prevalent cancer types. For example, HOX transcript antisense RNA (HOTAIR) expression is high in breast cancer tumors that are predisposed to metastasize, and the inhibition of HOTAIR expression blocks metastasis in mouse models (17). Metastasis associated lung adenocarcinoma transcript 1 (MALAT1) expression correlates with metastasis and overall survival rate in lung cancer (18). An increasing number of studies have explored methods to identify non-coding driver genes in cancers (19-21). Du et al (19) selected IncRNAs in recurrent somatic copy-number alterations (amplification) regions as candidate drivers. Knockdown of either prostate cancer-associated non coding RNA 1 (PCAN-R1) or 2 (PCAN-R2), which are the two most notably differentially expressed lncRNAs between normal prostate tissue and primary prostate cancer, resulted in substantial decreases in cell growth and colony formation in the androgen-dependent prostate cancer LNCaP cell line, suggesting that PCAN-R1 and PCAN-R2 have tumor-promoting functions in prostate cancer (19).

While numerous studies have investigated generalized variants $(7,9,11,12)$, this is not the case for cancer-specific somatic mutations. In the present study, a liver cancer-specific annotation, mainly from the ENCODE project, was used to investigate whether a combination of non-coding features may be predictive for non-coding pathogenic variants. This scoring system was then added to prioritize cancer-associated lncRNAs in liver cancer.

In a previous study, we successfully constructed a logistic regression model to score the functionalities of non-coding variants using an array of lung cancer-specific features (22). Considering the function impact of non-coding variants is largely feature and cancer type-dependent, in the present study, another logistic regression model was created based on a liver cancer-specific feature annotation to interpret the function information of non-coding pathogenic variants. Subsequently, this scoring system was applied to prioritize cancer-associated lncRNAs in liver cancer.

\section{Materials and methods}

Cancer mutation and pathogenic variant data. A total of 881,136 somatic mutations of human liver cancer were detected by whole-genome sequencing of 88 pairs of cancer and normal tissues. This data was obtained from the supplementary data files of the study by Alexandrov et al (23). Recurrent cancer mutation was defined as mutations that are recurrently mutated at least two times at the same site across multiple samples. Non-recurrent mutation denotes mutations that only occur once in all patients. In total, 1,121 mutations of liver cancer were defined as recurrent and the remaining mutations were considered to be non-recurrent. Germline polymorphism data comprising 38,248,779 single nucleotide polymorphisms (SNPs) was downloaded from the 1000 Genome project pilot 1 (24). SNPs with a derived allele frequency $\leq 0.01$ were considered neutral SNPs. Rare SNPs are the SNPs with an allele frequency $<0.01$. Disease-associated variants data contained in ClinVar and the human gene mutation database (HGMD) are published gene variants responsible for human inherited diseases $(25,26)$. Genome-wide association study (GWAS) SNPs from GWAS (27) are numerous common genetic variants associated with a trait or disease.

Genome-wide feature sets. Human genome annotations were obtained from Gencode (28), including protein coding genes, exons, introns, lncRNAs, lncRNA exons, introns, untranslated regions (UTRs) and non-coding exons (ncExon) (28). The 5' splicing sites are 10 nucleotides from the 5' end of the introns of genes. The $3^{\prime}$ splicing sites are 50 nucleotides from the $3^{\prime}$ end of the introns of genes (29). Evolutionarily conserved bases were identified using a recently published analysis of 46 mammalian genomes (30). Genome-wide phastCons scores were obtained from the study by Siepel et al (31). Sensitive regions from the study by Khurana et al (9) consist of binding sites or motifs of important transcription factors and contain a higher fraction of rare SNPs. Evolutionarily conserved structures (ECSs) from the study by Smith et al (32) are RNA secondary structures predicted using comparative structure prediction algorithms based on multiple genomes. Promoters, which are regions $2.5 \mathrm{~kb}$ from the transcription start sites, were generated by the Gerstein lab and are publicly available for download (9). RNA sequencing (RNA-seq) data in bam format, transcription factor binding sites (TFBSs), DNase I hypersensitive sites (DNase I), histone modification data, including $\mathrm{H} 3 \mathrm{~K} 4 \mathrm{me} 1$ and $\mathrm{H} 3 \mathrm{~K} 9 \mathrm{ac}$, of the Hepg2 cell line were acquired from ENCODE (33). Conserved TFBSs are transcription factor binding sites conserved in the human/mouse/rat alignment and obtained from UCSC directly (30). The expression level was calculated by counting the number of reads per $\mathrm{kb}$ per million reads (RPKM) for each protein coding gene and lncRNA. Genes with a RPKM $>20$ or $<0.25$ were defined as high and low-expressed regions, respectively. A wavelet-smoothed, weighted average signal, with high and low values that indicate early and late replication during the 
S phase, respectively (33), was used in the present study. Genome-wide replication timing was mapped to protein coding genes and lncRNAs, and an early-to-late ratio was calculated for each protein coding gene and lncRNA, as follows: Early-to-late ratio $=(\mathrm{G} 1 \mathrm{~b}+\mathrm{S} 1) /(\mathrm{S} 4+\mathrm{G} 2)$. As the early-to-late ratio is $>1$, genes are considered early replicated, while late replicated genes have an early-to-late ratio $<1$.

Cancer lncRNAs, consisting of 25 lncRNAs, are a curation of mammalian long non-coding transcripts that have been experimentally shown to be associated with a variety of cancer types (22). A list of cancer census genes was obtained from the current release of catalogue of somatic mutations in cancer (COSMIC) v71 (34).

Logistic regression model. The disease-implicated set of variants was composed of all pathogenic variants from the ClinVar and HGMD databases. Subsequent to the removal of coding variants, a set of 19,153 non-coding disease-implicated single nucleotide variants (SNVs) remained. For the control sets, neutral variants with a minor allele frequency $\geq 1 \%$ were used to reduce the possibility of including functional rare SNPs. A total of $15,789,242$ potential control SNVs were included in the model. In the logistic regression model, a matrix of 395,279 rows was formed throughout the non-coding genome; each row represents one type of combination of features. A disease-implicated set of variants was used as success (disease-causing variant), neutral SNPs were used as control, and the 26 genomic binary features were used as the predictor variable. The logistic regression model was constructed using a general linear model, and the receiver operating characteristic (ROC) curve was generated using a script in $\mathrm{R}$ generated by the present authors. The scores were predicted using the model for GWAS, neutral SNPs, non-recurrent and recurrent somatic mutations of liver cancer and then scaled using the following formula: Scaled score $=\log \left(\right.$ predicted score $\left.\mathrm{x} 10^{6}\right)$.

Prioritization of cancer-associated lncRNA candidates. In order to filter out potential functional lncRNAs involved in liver cancer, two different strategies were utilized. First, the fraction of high scoring regions and the average score for each lncRNA were calculated. Secondly, the top $10 \%$ of lncRNAs, which contained the highest fraction of high-scoring regions, and the $10 \%$ lncRNAs that had the highest average score were determined, and a subset of overlapping lncRNAs were generated by intersection of the two different lncRNAs sets, forming 847 functional lncRNA candidates, and the remaining lncRNAs were considered to be control lncRNAs.

RNA-seq data processing and expression analyses. The RNA-seq data of 12 pairs of liver cancer samples were obtained from the study by Zhang et al (GSE63863) (35). The reads were aligned to the hg19 genome using TopHat2 version 2.0.13 (36). Read counts were calculated with BEDTools v2.22.1 for each lncRNA (37). The relative expression level was calculated as the RPKM +1 and then log scaled for each IncRNA. DESeq2 Release (3.0) (38) was used to identify differentially expressed transcripts between tumor and normal pairs, with a false discovery rate (FDR) cutoff of $\leq 10^{-4}$ and absolute fold change cutoff of $\geq 1.5$.
Statistical analyses. The data were expressed as the mean values. The difference between groups was tested using the two-sided Mann-Whitney rank sum test (wilcox.test) or Fisher's exact test (fisher.test) in $\mathrm{R} . \mathrm{P}<0.05$ was considered to indicate a statistically significant difference.

\section{Results}

Logistic regression model successfully discriminates between functional non-coding variants and neutral variants. Density of disease-causing variants estimates showed that different features exhibit differential enrichment of deleterious variants (Fig. 1A). Conserved regions, conserved TFBS, UTRs, high-expressed regions and promoters showed the highest densities of disease-causing variants. By contrast, H3K9me3, late-replicated regions, ECSs, $\mathrm{H} 2 \mathrm{az}$ and $\mathrm{H} 3 \mathrm{~K} 27 \mathrm{me} 3$ are the least enriched features in disease-causing variants, indicating that various non-coding features have varied importance to the functionalities of non-coding variants. The present study used a logistic regression model to build a classifier to discriminate between the disease-associated and control variants. The present study analyzed the features that contribute most to the discriminative power of the present model (Fig. 1B). Generally, the present study observed that conserved regions, early-replicated regions, promoters, H3K36me3 and conserved TFBSs are the most positive factors contributing to the model, while H3K9me3, H3K79me2, H4K20me1 and ncExon are the most negative factors affecting the prediction capability of the model. The ROC curve for the classifier is shown in Fig. 1C. The area under the ROC curve (AUC) is 0.92, which demonstrates that the present model may discriminate between disease-implicated and control variants with a high specificity and sensitivity.

To establish whether the present prediction scores are likely to be generalizable to other data sets, the current study conducted experiments that demonstrate how the predicted scores may be applied to prioritize candidate functional variants. For the first experiment, non-coding variants associated with complex disease from genome-wide association studies (GWAS) were annotated. It was found that non-coding GWAS SNVs had a significantly higher average score compared with control variants (mean score, 5.8642 vs. 5.4707; $\mathrm{P}<0.001$; two-sided Mann-Whitney U test; Fig. 1D).

Recurrence is considered to be one potential sign of positive selection among tumors and is more likely to be associated with driver events (33). As an application to cancer studies, the present study annotated non-coding somatic mutations identified in whole-genome sequencing studies from 88 liver cancer samples. The present study identified recurrent somatic mutations that had occurred at the same site in multiple samples ( $n=1,121$ mutations) and found that these recurrent mutations were assigned a significantly higher average score compared with non-recurrent mutations (5.4101 vs. 5.2768; $\mathrm{P}=0.0125$, Mann-Whitney U test; Fig. 1D). This finding demonstrates that this approach may be useful in the detection of cancer driver mutations in liver cancer.

High-scoring regions define cancer 'hotspots' in introns, UTRs and lncRNAs. The present study defined 100-mb non-coding regions that had the highest scores predicted by the model as high-scoring regions, and gene features with the highest 
A

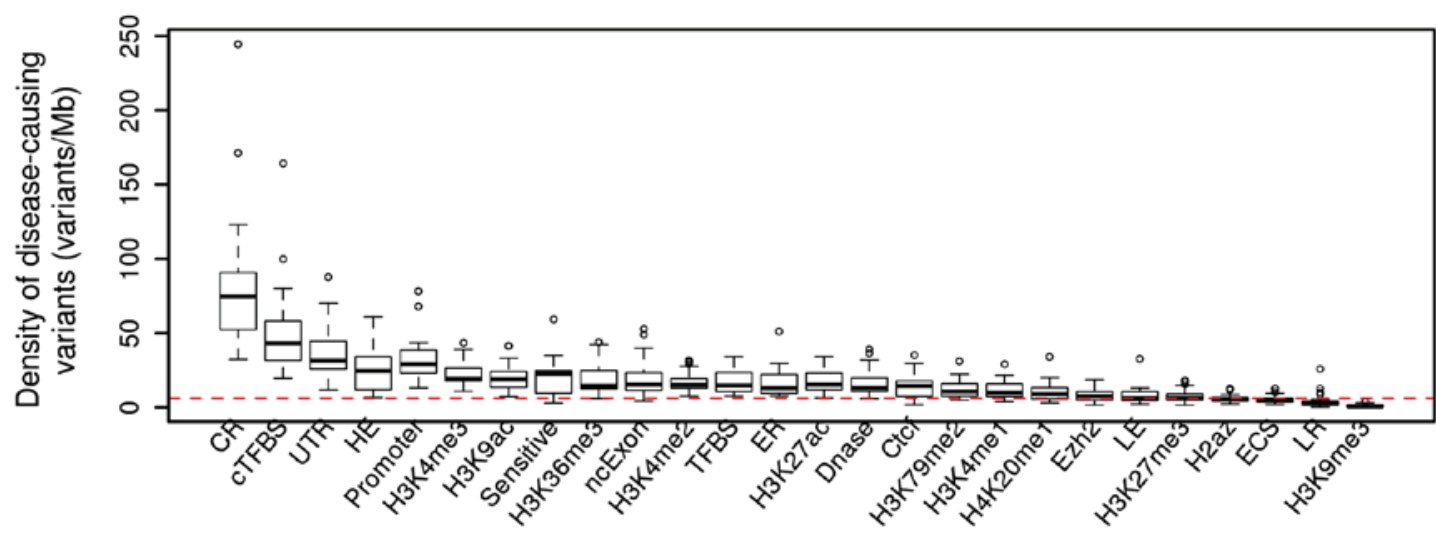

B

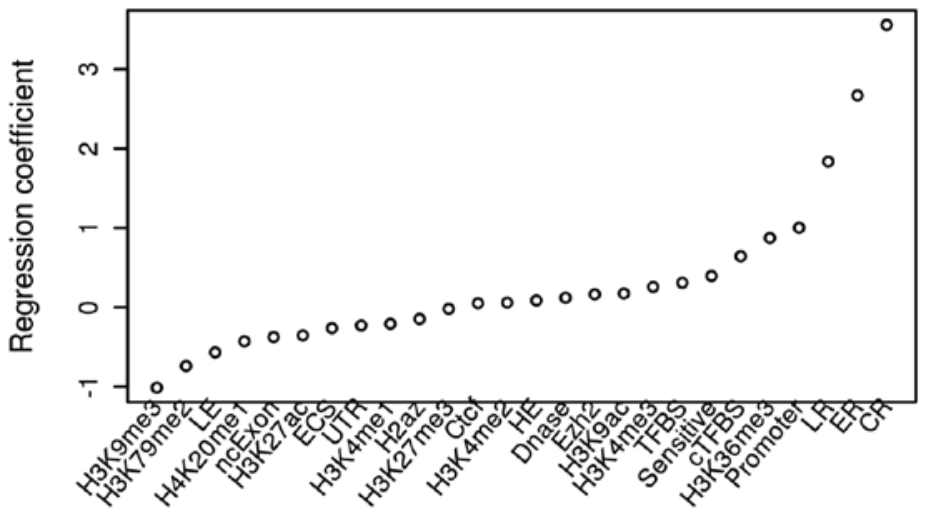

C

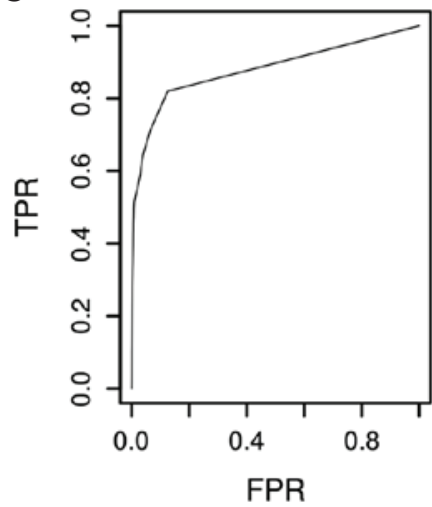

D

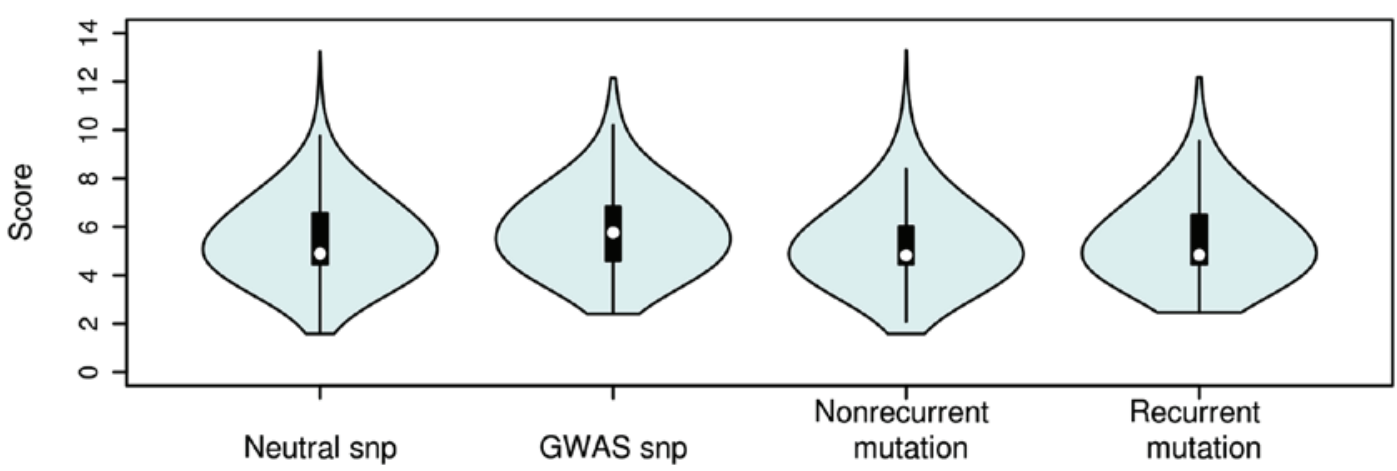

Figure 1. (A) Density of disease-causing variants from the ClinVar database and human gene mutation database associated with different genome features (red line, the average in the human genome). Different features exhibit differential enrichment of deleterious variants, with conserved regions highest and H3K9me3 lowest. (B) Regression coefficient for each feature. (C) Receiver operating characteristic curve for the logistic regression model. (D) Predicted scores for GWAS, neutral SNPs, and non-recurrent and recurrent somatic mutations of liver cancer. The scores were scaled using the formula 'scaled score $=$ log (predicted score $\left.\mathrm{x}^{6} 0^{6}\right)^{\prime}$. GWAS disease associated variants and recurrent somatic mutations of liver cancer showed elevated average scores as compared with neutral SNPs and non-recurrent somatic mutations respectively. TFBS, transcription factor binding site; cTFBS, conserved TFBS; UTR, untranslated region; CR, conserved region; SNP, single nucleotide polymorphism; Sensitive, known regions with a high ratio of rare SNP (allele frequency <0.01); ER, early replicated regions; LR, late replicated regions; HE, high expressed regions; LE, low expressed regions; ECS, evolutionarily conserved structure; Dnase I, Dnase I hypersensitive site; H3K/H4K, histone modification data; ncExon, non-coding exon; TPR, true positive rate; FPR, false positive rate.

fractions of high-scoring regions were sought. Features with the highest fractions of high scoring regions included intronic 5' and 3' splice sites and 3'-UTRs (Fig. 2A). Splicing sites were scored significantly higher compared with adjacent intronic regions in protein coding genes (9.3463 vs. 8.3263; $\mathrm{P}<0.01$; Fig. 2B) and lncRNAs (8.2544 vs. 7.9248; P<0.001; Fig. 2B). Furthermore, known cancer genes from COSMIC have significantly more high-scoring regions in the gene introns and UTRs compared with non-cancer genes (0.0850 vs. 0.0640 ; $\mathrm{P}<0.001$; Fig. 2C). In general, lncRNAs do not contain a large fraction of high-scoring regions (Fig. 2C). However, the known cancer-associated lncRNAs showed a significantly increased fraction of high-scoring regions as compared to general IncRNAs (0.0997 vs. 0.0404; $\mathrm{P}<0.001$; Fig. 2C). For example, HOTAIR and MALAT1 are among the top $10 \%$ of lncRNAs with respect to overlap with high-scoring regions (Fig. 2D).

Prioritization of liver cancer-associated lncRNAs with the scoring system. In the present study, 847 lncRNAs were identified by combining the fraction of high-scoring regions and 


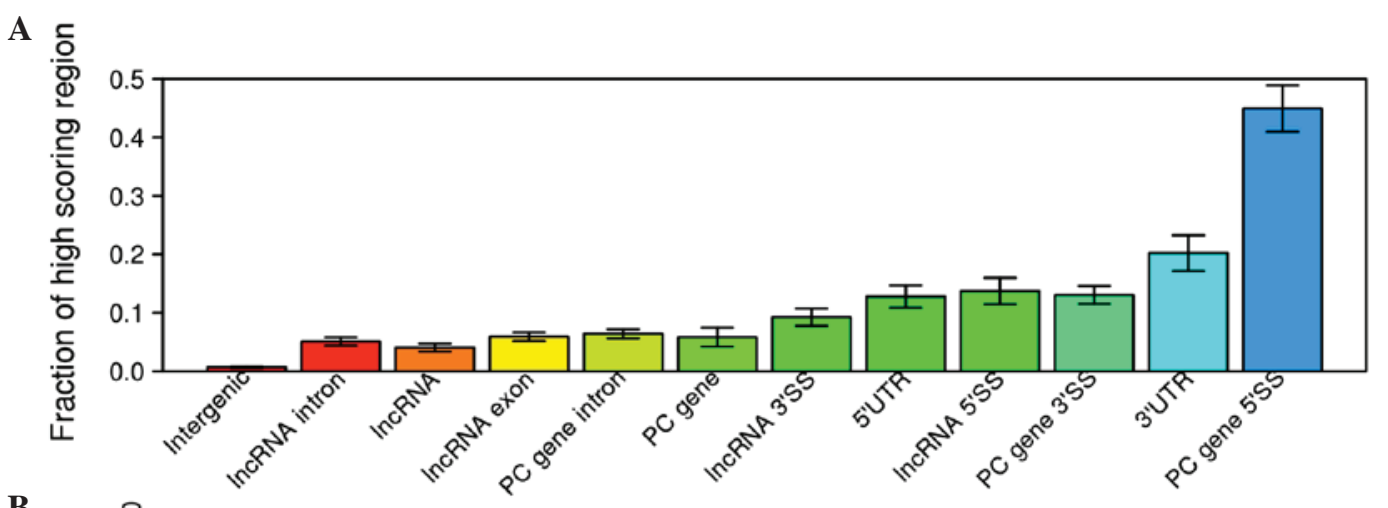

B

(5)

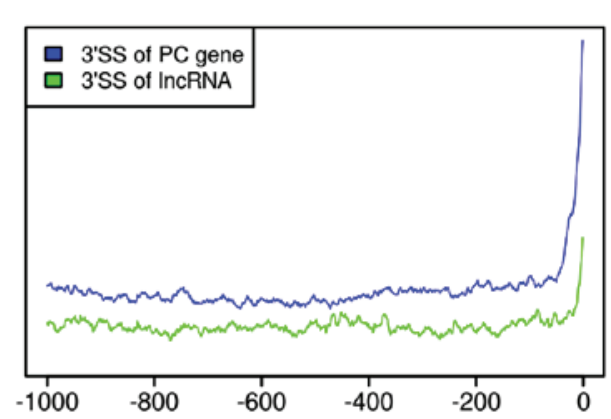

Distance from splicing sites (bp)

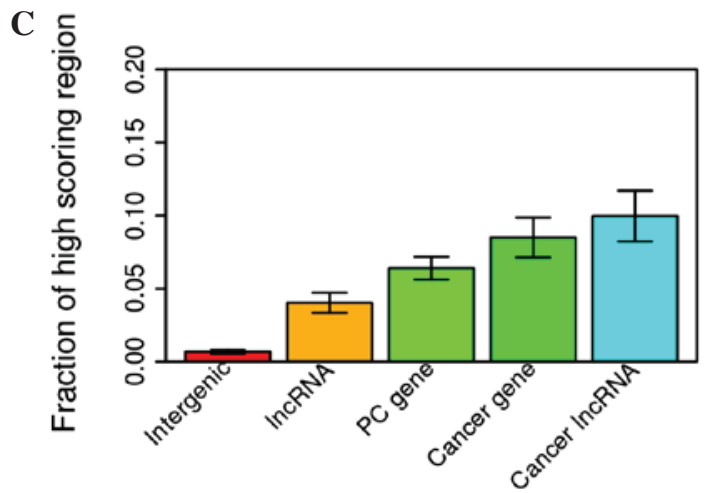

D

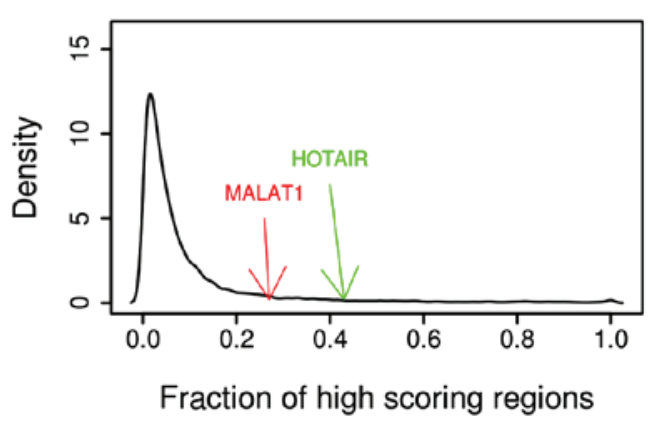

Figure 2. Characterization of high-scoring genome regions in liver cancer. (A) Fraction of high-scoring genome regions in various non-coding features. Features with the highest fractions of high-scoring regions included intronic 5' and 3' splice sites and $3^{\prime}-\mathrm{UTR}$. (B) Average score in protein-coding gene and lncRNA introns near the 5'-splice site (left) and 3'-splice site (right). The scores were predicted by the model and scaled using the formula 'scaled score $=$ log (predicted score $\left.\mathrm{x} 10^{6}\right)^{\prime}$. Splicing sites were scored significantly higher compared to adjacent intronic regions in protein coding genes and lncRNAs (C) Fraction of high scoring regions in different gene classes. Known cancer genes from COSMIC and cancer associated lncRNAs had an increased fraction of high-scoring regions as compared to non-cancer genes and general lncRNAs respectively. (D) Kernal density plot of fraction of high-scoring regions in lncRNAs; HOTAIR and MALAT1 are two exemplary cancer lncRNAs that contain high coverage of high-scoring regions. Distribution of fraction of high-scoring regions in lncRNAs. IncRNA, long non-coding RNS; PC gene, protein coding gene; 5'SS, 5' splicing site 10 nucleotides from the 5'-end of introns of genes; 3'SS, 3 ' splicing site 50 nucleotides from the 3'-end of introns of genes; UTR, untranslated region; MALAT1, metastasis associated lung adenocarcinoma transcript 1; HOTAIR, HOX transcript antisense RNA.

the average score of lncRNAs. Among these are lncRNAs that are known to be cancer-associated, such as MALAT1, HOTAIR and growth arrest specific 5 (GAS5). This final subset of IncRNAs was found to be significantly more conserved compared with other control lncRNAs, in terms of fraction of conserved regions ( 0.1635 vs. $0.0536 ; \mathrm{P}<0.001$; Fig. $3 \mathrm{~A})$ and phastCons score ( 0.2813 vs. 0.2644 ; $\mathrm{P}<0.001$; Fig. $3 \mathrm{~B}$ ). It was also observed that this subset of IncRNAs had a lower somatic mutation density compared with the control lncRNAs (219.4753 vs. 329.7922 mutations/Mb; P<0.001; Fig. 3C); RNA-seq data of 12 pairs of liver cancer samples were obtained from the study by Zhang et al (35). Read alignment was conducted using TopHat 2 and coverage was calculated for each IncRNAs using BEDTools. It was found that the list of lncRNA candidates had increased average expression levels compared with the control lncRNAs in cancer and normal samples [log scaled (RPKM+1), 1.3868 vs. 0.6327 ; P $<0.001$; Fig. 3D]; DESeq2 was used to evaluate the different expression of lncRNAs in 12 pairs of liver cancer and adjacent normal samples. IncRNAs with FDR $\leq 10^{-4}$ and absolute fold change $\geq 1.5$ were considered to be differentially expressed. In total, there were $353 \mathrm{lncRNAs}$ that met the selection criteria, 23 of which are among the list of potentially cancer-associated lncRNAs (Fig. 4).

\section{Discussion}

It is evident that only a small subset of genetic variations contributes to tumor evolution by providing cells with a 
A

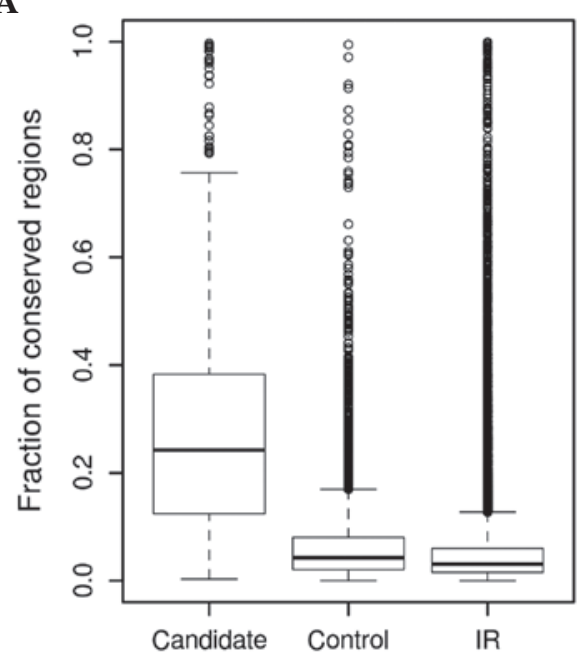

C

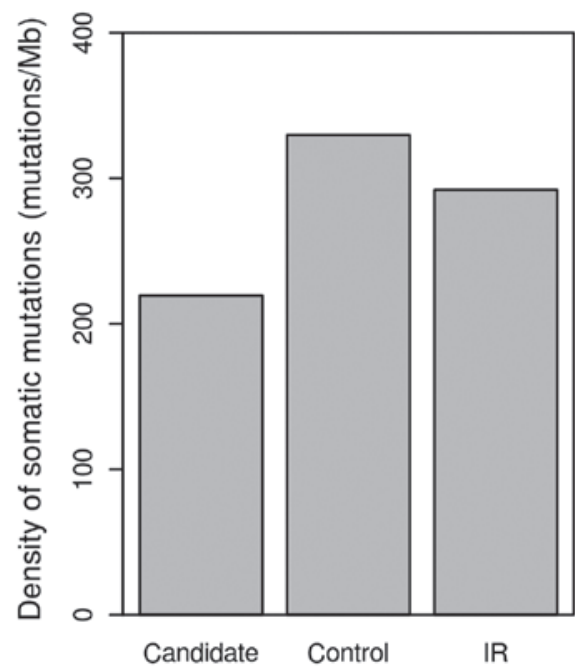

B

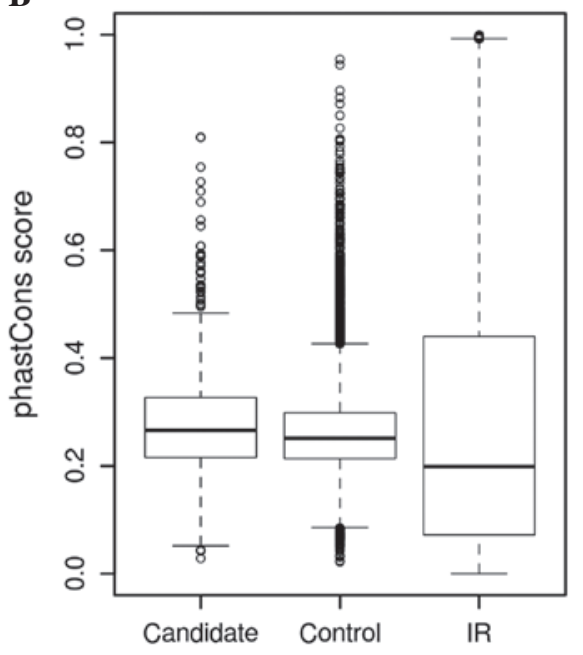

D

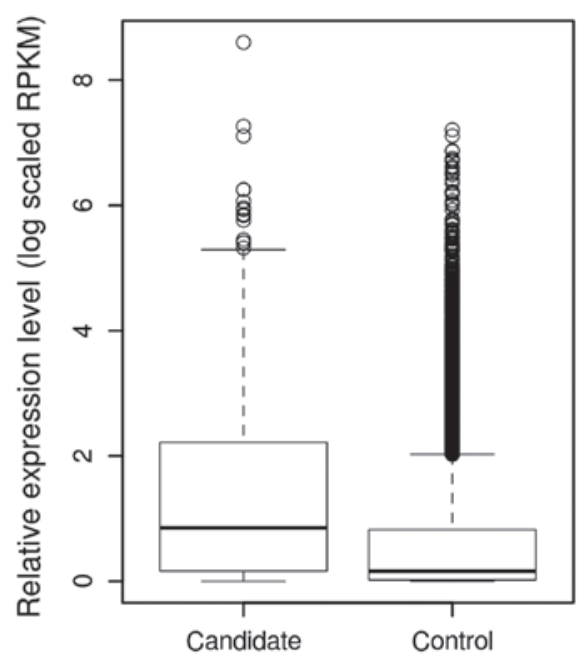

Figure 3. Characterization of functional candidates in liver cancer. (A) Fraction of conserved regions in functional candidates, controls and IRs; functional candidates contain increased enrichment of conserved regions in comparison with controls and IRs. (B) Average phastCons scores for functional candidates, controls and IRs; functional candidates show higher average phastCons scores than controls and IRs. (C) Average densities of somatic mutations for functional candidates, controls and IRs; functional candidates are less frequently mutated compared with controls and IRs. (D) Relative expression [log scaled $(\mathrm{RPKM}+1)$ ] for functional candidates, controls and IRs; functional candidates are overexpressed compared with controls and IRs. lncRNA, long non-coding RNA; candidate, lncRNA candidate; control, control lncRNA; IR, intergenic regions; RPKM, reads per kb per million reads.
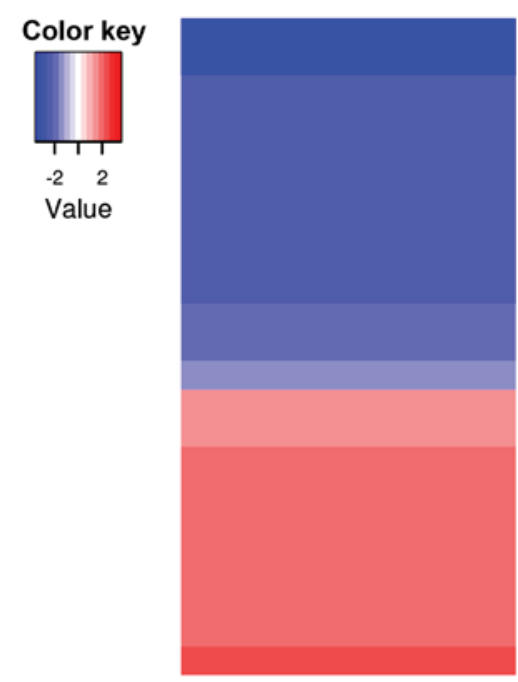

Figure 4. Expression profile for 23 differentially expressed long non-coding RNA candidates.

selective advantage over their neighbors (10). The damaging impact of coding mutations may be evaluated efficiently with a variety of tools; however, functional annotation of mutations in the non-coding fraction of the human genome is markedly more obscure and challenging.

Recently, GWAVA used all variations annotated as 'regulatory mutations' from the public release of HGMD and combined annotations to build three random forest classifiers that prioritize disease-associated variants (12); however, this study focused on regulatory mutations of the HGMD database and predicted regulatory pathogenic variants, which was incomplete and limited in scope to the regulatory regions. The present logistic regression model included all non-coding pathogenic variants from HGMD and ClinVar databases, which allows for evaluation of damaging impact of any variant in the non-coding genome. Furthermore, a liver cancer-specific annotation was used, which facilitated the identification of driver mutations in a liver cancer-specific fashion. It was 
found that non-coding features, such as conserved regions, early replicated regions, promoter, $\mathrm{H} 3 \mathrm{~K} 36 \mathrm{me} 3$, conserved TFBS, sensitive regions, TFBS, H3K4me 3 and $\mathrm{H} 3 \mathrm{~K} 9 \mathrm{ac}$ are among the positive factors that most contribute to the model. Histone modifications, such as $\mathrm{H} 3 \mathrm{~K} 4 \mathrm{me} 3$ and $\mathrm{H} 3 \mathrm{~K} 9 \mathrm{ac}$, are hallmarks of actively transcribed protein-coding promoters in eukaryotes, and H3K36me3 has long been associated with the gene bodies of actively transcribed genes (40). All these findings support the hypothesis that conserved regions and regulatory elements play notable roles in the formation and functionality of pathogenic variants in the non-coding genome.

The AUC demonstrates how well a classifier can discriminate between disease and control variants; the AUC of the present logistic regression model was as high as 0.92 , which showed a reliable and high-efficient performance. Furthermore, the present study showed the utility of the present model by providing two types of examples of common experiments using disease or trait-associated GWAS variants and recurrent cancer mutations. The present study demonstrates that the present model effectively discriminates non-coding GWAS SNVs from control variants. Recurrence of somatic mutations is a widely used proxy of likely function; the present scoring system scored recurrent mutations significantly higher compared with non-recurrent mutations, suggesting this approach may allow for the identification of cancer driver mutations.

With respect to the distribution of high scoring regions identified by the present model, it was found that splicing sites of either protein coding genes or lncRNAs and UTRs were most enriched with the highest fraction of high scoring regions, as these regions are highly evolutionarily conserved across mammals (41). Notably, it was found that known cancer genes and cancer-implicated lncRNAs contain a higher fraction of high-scoring regions compared with non-cancer-associated counterparts. Two typical examples are MALAT1 and HOTAIR, which have been involved in the tumorigenesis and progression in a variety of cancer types $(17,18,42-47)$. The fraction of high scoring regions and average score predicted by the present model was combined for each lncRNA and filtered out the a subset of functional lncRNA candidates, which include experimentally characterized functional lncRNAs, such as MALAT1, HOTAIR, HOXA transcript antisense RNA, myeloid-specific 1 and GAS5. The present study found that this small subset of lncRNAs are more conserved, less mutated and demonstrate increased expression compared with the control lncRNAs, and 23 of the 847 lncRNAs identified are differentially expressed in 12 pairs of liver cancer and normal samples. These lncRNAs are notable candidates for experimental validation and characterization in future studies.

Overall, the present study defined a scoring system for evaluating the damaging effect of non-coding variants in liver cancer. This system allows the identification of putative harmful mutations in a liver-cancer specific fashion in the introns and UTRs of mRNAs, as well as prioritizing a number of lncRNA candidates for additional experimental validation.

\section{Acknowledgements}

The present study was supported by the National Natural Sciences Foundation of China (grant no, 81272142; to Xin LV).

\section{References}

1. Robison K: Application of second-generation sequencing to cancer genomics. Brief Bioinform 11: 524-534, 2010.

2. Greenman C, Stephens P, Smith R, Dalgliesh GL, Hunter C, Bignell G, Davies H, Teague J, Butler A, Stevens C, et al: Patterns of somatic mutation in human cancer genomes. Nature 446: 153-158, 2007.

3. Sim NL, Kumar P, Hu J, Henikoff S, Schneider G and Ng PC: SIFT web server: Predicting effects of amino acid substitutions on proteins. Nucleic Acids Res 40 (Web Server Issue): W452-W457, 2012.

4. Adzhubei IA, Schmidt S, Peshkin L, Ramensky VE, Gerasimova A, Bork P, Kondrashov AS and Sunyaev SR: A method and server for predicting damaging missense mutations. Nat Methods 7: 248-249, 2010.

5. ENCODE Project Consortium: An integrated encyclopedia of DNA elements in the human genome. Nature 489: 57-74, 2012.

6. Bernstein BE, Stamatoyannopoulos JA, Costello JF, Ren B, Milosavljevic A, Meissner A, Kellis M, Marra MA, Beaudet AL, Ecker JR, et al: The NIH roadmap epigenomics mapping consortium. Nat Biotechnol 28: 1045-1048, 2010.

7. Boyle AP, Hong EL, Hariharan M, Cheng Y, Schaub MA, Kasowski M, Karczewski KJ, Park J, Hitz BC, Weng S, et al: Annotation of functional variation in personal genomes using RegulomeDB. Genome Res 22: 1790-1797, 2012.

8. Ward LD and Kellis M: HaploReg: A resource for exploring chromatin states, conservation and regulatory motif alterations within sets of genetically linked variants. Nucleic Acids Res 40 (Database Issue): D930-D934, 2012.

9. Khurana E, Fu Y, Colonna V, Mu XJ, Kang HM, Lappalainen T, Sboner A, Lochovsky L, Chen J, Harmanci A, et al: Integrative annotation of variants from 1092 humans: Application to cancer genomics. Science 342: 1235587, 2013.

10. Li J, Drubay D, Michiels S and Gautheret D: Mining the coding and non-coding genome for cancer drivers. Cancer Lett 369: 307-315, 2015.

11. Kircher M, Witten DM, Jain P, O'Roak BJ, Cooper GM and Shendure J: A general framework for estimating the relative pathogenicity of human genetic variants. Nat Genet 46: 310-315, 2014.

12. Ritchie GR,Dunham I,Zeggini E and Flicek P: Functional annotation of noncoding sequence variants. Nat Methods 11: 294-296, 2014.

13. Fu Y, Liu Z, Lou S, Bedford J, Mu XJ, Yip KY, Khurana E and Gerstein M: FunSeq2: A framework for prioritizing noncoding regulatory variants in cancer. Genome Biol 15: 480, 2014.

14. Cooper GM, Stone EA, Asimenos G; NISC Comparative Sequencing Program, Green ED, Batzoglou S and Sidow A: Distribution and intensity of constraint in mammalian genomic sequence. Genome Res 15: 901-913, 2005.

15. Nie L, Wu HJ, Hsu JM, Chang SS, Labaff AM, Li CW, Wang Y, Hsu JL and Hung MC: Long non-coding RNAs: Versatile master regulators of gene expression and crucial players in cancer. Am J Transl Res 4: 127-150, 2012.

16. Gutschner T and Diederichs S: The hallmarks of cancer: A long non-coding RNA point of view. RNA Biol 9: 703-719, 2012.

17. Gupta RA, Shah N, Wang KC, Kim J, Horlings HM, Wong DJ, Tsai MC, Hung T, Argani P, Rinn JL, et al: Long non-coding RNA HOTAIR reprograms chromatin state to promote cancer metastasis. Nature 464: 1071-1076, 2010.

18. Ji P, Diederichs S, Wang W, Böing S, Metzger R, Schneider PM, Tidow N, Brandt B, Buerger H, Bulk E, et al: MALAT-1, a novel noncoding RNA and thymosin beta4 predict metastasis and survival in early-stage non-small cell lung cancer. Oncogene 22: 8031-8041, 2003.

19. Du Z, Fei T, Verhaak RG, Su Z, Zhang Y, Brown M, Chen Y and Liu XS: Integrative genomic analyses reveal clinically relevant long noncoding RNAs in human cancer. Nat Struct Mol Biol 20: 908-913, 2013.

20. Mularoni L, Sabarinathan R, Deu-Pons J, Gonzalez-Perez A and López-Bigas N: OncodriveFML: a general framework to identify coding and non-coding regions with cancer driver mutations. Genome Biol 17: 128, 2016.

21. Yan X, Hu Z, Feng Y, Hu X, Yuan J, Zhao SD, Zhang Y, Yang L, Shan W, He Q, et al: Comprehensive Genomic Characterization of Long Non-coding RNAs across Human Cancers. Cancer Cell 28: 529-540, 2015.

22. Li H and Lv X: Functional annotation of noncoding variants and prioritization of cancer-associated lncRNAs in lung cancer. Oncol Lett 12: 222-230, 2016. 
23. Alexandrov LB, Nik-Zainal S, Wedge DC, Aparicio SA, Behjati S, Biankin AV, Bignell GR, Bolli N, Borg A, Børresen-Dale AL, et al: Signatures of mutational processes in human cancer. Nature 500: 415-421, 2013.

24. 1000 Genomes Project Consortium; Abecasis GR, Auton A, Brooks LD, DePristo MA, Durbin RM, Handsaker RE, Kang HM, Marth GT and McVean GA: An integrated map of genetic variation from 1,092 human genomes. Nature 491: 56-65, 2012.

25. Landrum MJ, Lee JM, Riley GR, Jang W, Rubinstein WS, Church DM and Maglott DR: ClinVar: Public archive of relationships among sequence variation and human phenotype. Nucleic Acids Res 42 (Database Issue): D980-D985, 2014.

26. Stenson PD, Mort M, Ball EV, Howells K, Phillips AD, Thomas NS and Cooper DN: The human gene mutation database: 2008 update. Genome Med 1: 13, 2009.

27. Beck T, Hastings RK, Gollapudi S, Free RC and Brookes AJ: GWAS Central: A comprehensive resource for the comparison and interrogation of genome-wide association studies. Eur J Hum Genet 22: 949-952, 2014.

28. Harrow J, Frankish A, Gonzalez JM, Tapanari E, Diekhans M, Kokocinski F, Aken BL, Barrell D, Zadissa A, Searle S, et al: GENCODE: The reference human genome annotation for the ENCODE project. Genome Res 22: 1760-1774, 2012.

29. Ward AJ and Cooper TA: The pathobiology of splicing. J Pathol 220: 152-163, 2010.

30. Karolchik D, Barber GP, Casper J, Clawson H, Cline MS, Diekhans M, Dreszer TR, Fujita PA, Guruvadoo L, Haeussler M, et al: The UCSC Genome Browser database: 2014 update. Nucleic Acids Res 42 (Database Issue): D764-D770, 2014.

31. Siepel A, Bejerano G, Pedersen JS, Hinrichs AS, Hou M, Rosenbloom K, Clawson H, Spieth J, Hillier LW, Richards S, et al: Evolutionarily conserved elements in vertebrate, insect, worm and yeast genomes. Genome Res 15: 1034-1050, 2005.

32. Smith MA, Gesell T, Stadler PF and Mattick JS: Widespread purifying selection on RNA structure in mammals. Nucleic Acids Res 41: 8220-8236, 2013.

33. Rosenbloom KR, Sloan CA, Malladi VS, Dreszer TR, Learned K, Kirkup VM, Wong MC, Maddren M, Fang R, Heitner SG, et al: ENCODE data in the UCSC genome browser: Year 5 update. Nucleic Acids Res 41 (Database Issue): D56-D63, 2013.

34. Forbes SA, Bindal N, Bamford S, Cole C, Kok CY, Beare D, Jia M, Shepherd R, Leung K, Menzies A, et al: COSMIC: Mining complete cancer genomes in the catalogue of somatic mutations in cancer. Nucleic Acids Res 39 (Database Issue): D945-D950, 011.

35. Zhang H, Weng X, Ye J, He L, Zhou D and Liu Y: Promoter hypermethylation of TERT is associated with hepatocellular carcinoma in the Han Chinese population. Clin Res Hepatol Gastroenterol 39: 600-609, 2015
36. Kim D, Pertea G, Trapnell C, Pimentel H, Kelley R and Salzberg SL: TopHat2: Accurate alignment of transcriptomes in the presence of insertions, deletions and gene fusions. Genome Biol 14: R36, 2013.

37. Quinlan AR and Hall IM: BEDTools: A flexible suite of utilities for comparing genomic features. Bioinformatics 26: 841-842, 2010.

38. Love MI, Huber W and Anders S: Moderated estimation of fold change and dispersion for RNA-seq data with DESeq2. Genome Biol 15: 550, 2014.

39. Dees ND, Zhang Q, Kandoth C, Wendl MC, Schierding W, Koboldt DC, Mooney TB, Callaway MB, Dooling D, Mardis ER, et al: MuSiC: Identifying mutational significance in cancer genomes. Genome Res 22: 1589-1598, 2012.

40. Hon GC, Hawkins RD and Ren B: Predictive chromatin signatures in the mammalian genome. Hum Mol Genet 18: R195-R201, 2009.

41. Washietl S, Kellis M and Garber M: Evolutionary dynamics and tissue specificity of human long noncoding RNAs in six mammals. Genome Res 24: 616-628, 2014.

42. Yang MH, Hu ZY, Xu C, Xie LY, Wang XY, Chen SY and Li ZG: MALAT1 promotes colorectal cancer cell proliferation/migration/invasion via PRKA kinase anchor protein 9. Biochim Biophys Acta 1852: 166-174, 2015.

43. Shen L, Chen L, Wang Y, Jiang X, Xia H and Zhuang Z: Long noncoding RNA MALAT1 promotes brain metastasis by inducing epithelial-mesenchymal transition in lung cancer. J Neurooncol 121: 101-108, 2015.

44. Okugawa Y, Toiyama Y, Hur K, Toden S, Saigusa S, Tanaka K, Inoue $\mathrm{Y}$, Mohri $\mathrm{Y}$, Kusunoki M, Boland CR and Goel A: Metastasis-associated long non-coding RNA drives gastric cancer development and promotes peritoneal metastasis. Carcinogenesis 35: 2731-2739, 2014.

45. Han Y, Liu Y, Zhang H, Wang T, Diao R, Jiang Z, Gui Y and Cai Z: Hsa-miR-125b suppresses bladder cancer development by down-regulating oncogene SIRT7 and oncogenic long non-coding RNA MALAT1. FEBS Lett 587: 3875-3882, 2013.

46. Deng Q, Sun H, He B, Pan Y, Gao T, Chen J, Ying H, Liu X, Wang F, Xu Y and Wang S: Prognostic value of long non-coding RNA HOTAIR in various cancers. PLoS One 9: e110059, 2014.

47. Endo H, Shiroki T, Nakagawa T, Yokoyama M, Tamai K, Yamanami H, Fujiya T, Sato I, Yamaguchi K, Tanaka N, et al: Enhanced expression of long non-coding RNA HOTAIR is associated with the development of gastric cancer. PLoS One 8: e77070, 2013. 